IJMMS 25:10 (2001) 655-668

PII. S0161171201003891

http://ijmms.hindawi.com

(C) Hindawi Publishing Corp.

\title{
a-MINIMAL SETS AND RELATED TOPICS IN TRANSFORMATION SEMIGROUPS (II)
}

\author{
MASOUD SABBAGHAN and FATEMAH AYATOLLAH ZADEH SHIRAZI
}

(Received 20 January 1999 and in revised form 12 September 1999)

\begin{abstract}
We give some generalizations of proximal relation and distal structure relation of a transformation semigroup in terms of $A$-minimal sets and $A$-minimal sets instead of minimal right ideals and conclude similar results.
\end{abstract}

2000 Mathematics Subject Classification. Primary 54H15.

1. Preliminaries. By a transformation semigroup $(X, S, \rho)$ (or simply $(X, S)$ ) we mean a compact Hausdorff topological space $X$, a discrete topological semigroup $S$ with identity $e$, and a continuous map $\rho: X \times S \rightarrow X(\rho(x, s)=x s \forall x \in X, \forall s \in S)$, such that

(1) $x e=x \forall x \in X$

(2) $x(s t)=(x s) t \forall x \in X, \forall s, t \in S$.

In the transformation semigroup $(X, S)$, for each $s \in S$ define $\pi^{s}: X \rightarrow X$ by $\pi^{s}(x)=$ $x s(\forall x \in X)$. We assume the semigroup $S$ acts effectively on $X$, that is, for each $s, t \in S, s \neq t$ if and only if $\pi^{s} \neq \pi^{t}$. The closure of $\left\{\pi^{s} \mid s \in S\right\}$ in $X^{X}$ (with pointwise convergence topology) is called the enveloping semigroup (or Ellis semigroup) of $(X, S)$ and is denoted by $\mathrm{E}(X, S)$ (or simply $\mathrm{E}(X)$ ), $\mathrm{E}(X)$ has a semigroup structure [1]. A nonempty subset $I$ of $\mathrm{E}(X)$ is called a right ideal of $\mathrm{E}(X)$ if $I \mathrm{E}(X) \subseteq I$, moreover, if the right ideal $I$ of $\mathrm{E}(X)$ does not have any proper subset which is a right ideal of $\mathrm{E}(X)$, then $I$ is called a minimal right ideal of $\mathrm{E}(X)$, the set of all minimal right ideals of $\mathrm{E}(X)$ is denoted by $\operatorname{Min}(\mathrm{E}(X))$. An element $u$ of $\mathrm{E}(X)$ is called idempotent if $u^{2}=u$. For $p \in \mathrm{E}(X)$ and $a \in X$ the maps $\mathrm{L}_{p}: \mathrm{E}(X) \rightarrow \mathrm{E}(X)$ and $\theta_{a}: \mathrm{E}(X) \rightarrow X$ defined by $\mathrm{L}_{p}(q)=p q$ and $\theta_{a}(q)=a q(q \in \mathrm{E}(X))$, respectively, are continuous [2, Propositions 3.2 and 3.3]. Let $I$ be a right ideal of $\mathrm{E}(X), B \subseteq \mathrm{E}(X), C \subseteq X(B, C \neq \varnothing)$ and $a \in X$. Standing notations:

$$
\begin{array}{rlrl}
\mathrm{S}(I) & =\left\{p \in I \mid \mathrm{L}_{p}: I \rightarrow I \text { is surjective }\right\}, & F(a, B)=\{p \in B \mid a p=a\}, \\
\mathrm{I}(I) & =\left\{p \in I \mid \mathrm{L}_{p}: I \rightarrow I \text { is injective }\right\}, & F(C, B)=\bigcap_{c \in C} F(c, B), \\
\mathrm{B}(I) & =\left\{p \in I \mid \mathrm{L}_{p}: I \rightarrow I \text { is bijective }\right\}, & \bar{F}(C, B)=\{p \in B \mid C p=C\}, \\
J(B) & =\left\{u \in B \mid u^{2}=u\right\} .
\end{array}
$$

A nonempty subset $Z$ of $X$ is called invariant if $Z S \subseteq Z$, moreover, a closed invariant subset $Z$ of $X$ is called minimal if it does not have any proper closed invariant subset. Also $a \in X$ is called almost periodic if $\overline{a S}=a \mathrm{E}(X)$ is a minimal subset of $X$ [3, Theorems 1.15 and 1.17]. Let $K$ be a closed right ideal of $\mathrm{E}(X)$, then $K$ is called 
an $A$-minimal set if for each $b \in A, b K=b \mathrm{E}(X)$ and $K$ does not contain any closed right ideal $L$ of $\mathrm{E}(X)$ such that $K \neq L$ and for each $b \in A, b L=b \mathrm{E}(X)$, also $K$ is called an $A$ - $\overline{\text { minimal }}$ set if $A K=A \mathrm{E}(X)$ and $K$ does not contain any closed right ideal $L$ of $\mathrm{E}(X)$ such that $K \neq L$ and $A L=A \mathrm{E}(X)$; the collection of all $A$-minimal sets is denoted by $\overline{\mathrm{M}}_{(X, S)}(A)$ or simply $\overline{\mathrm{M}}(A)$ and the collection of all $A$ - $\overline{\text { minimal }}$ sets is denoted by $\overline{\overline{\mathrm{M}}}_{(X, S)}(A)$ or simply $\overline{\overline{\mathrm{M}}}(A)$; we use $\mathrm{M}_{(X, S)}(a)$ (or simply $\mathrm{M}(a)$ ) instead of $\overline{\mathrm{M}}_{(X, S)}(\{a\})$ and its elements are called $a$-minimal sets; in addition we introduce the following sets:

$$
\begin{aligned}
& \bar{M}(X, S)=\{D \subseteq X \mid D \neq \varnothing, \forall K \in \overline{\mathrm{M}}(D) J(F(D, K)) \neq \varnothing\}, \\
& \overline{\bar{M}}(X, S)=\{D \subseteq X \mid D \neq \varnothing, \overline{\overline{\mathrm{M}}}(D) \neq \varnothing, \forall K \in \overline{\overline{\mathrm{M}}}(D) J(F(D, K)) \neq \varnothing\},
\end{aligned}
$$

the transformation semigroup $(X, S)$ is called $A \stackrel{(-)}{ }$ distal (or simply $A$-distal) if for each

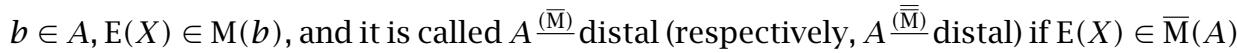
(respectively, $\mathrm{E}(X) \in \overline{\overline{\mathrm{M}}}(A)$ ).

Let $(X, S)$ and $(Y, S)$ be transformation semigroups, then the continuous map $\varphi$ : $(X, S) \rightarrow(Y, S)$ is called a homomorphism if $\varphi(x s)=\varphi(x) s(\forall x \in X, \forall s \in S)$, if $\varphi$ is onto, then there exists a unique induced homomorphism $\hat{\varphi}:(\mathrm{E}(X), S) \rightarrow(\mathrm{E}(Y), S)$ which is onto and for each $x \in X$, the following diagram commutes:

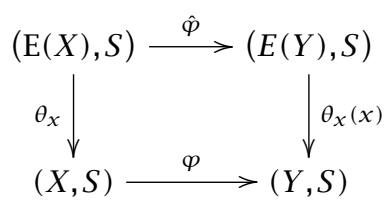

moreover, $\hat{\varphi}$ is a semigroup homomorphism; if $\varphi$ is onto and one-to-one, it is called an isomorphism, and $\hat{\varphi}$ is an isomorphism too [2, Proposition 3.8]. An equivalence relation $\mathfrak{R}$ on $X$ is called invariant if $\mathfrak{R}$ is an invariant subset of the transformation semigroup $(X \times X, S)$. Let $\mathfrak{R}$ be an equivalence relation on $X$, then $\pi_{\mathfrak{R}}: X \rightarrow X / \mathfrak{R}$ $\left(\pi_{\mathfrak{R}}(x)=[x]_{\mathfrak{R}}(\forall x \in X)\right)$ is the natural canonical map.

For the remainder of this paper $(X, S)$ is a fixed transformation semigroup, with $e$ as the identity element of $S$ and $\Delta_{A}=\{(x, x) \mid x \in A\}$.

DeFinition 1.1. Let $A$ be a nonempty subset of $X$ and let

$\mathfrak{I}=\{\mathfrak{R} \mid \mathfrak{R}$ is a closed invariant equivalence relation on $X$ such that $(X / \mathfrak{R}, S)$ is distal $\}$,

$\mathfrak{J}_{0}=\{\mathfrak{R} \mid \mathfrak{R}$ is a closed invariant equivalence relation on $X$ such that $(X / \mathfrak{R}, S)$ is $[A]_{\mathfrak{R}}$-distal $\}$,

$\mathfrak{J}_{1}=\{\mathfrak{R} \mid \mathfrak{R}$ is a closed invariant equivalence relation on $X$ such that $(X / \mathfrak{R}, S)$ is $[A]_{\mathfrak{K}} \frac{(\overline{\mathrm{M}})}{\mathrm{distal}\}}$,

$\mathfrak{J}_{2}=\{\mathfrak{R} \mid \mathfrak{R}$ is a closed invariant equivalence relation on $X$ such that $(X / \mathfrak{R}, S)$ is $[A]_{\mathfrak{R}} \frac{(\overline{\overline{\mathrm{M}}})}{\operatorname{distal}\}}$, 
then $\bigcap_{\mathfrak{R} \in \mathfrak{I}} \mathfrak{R}, \bigcap_{\mathfrak{R} \in \mathfrak{I}_{0}} \mathfrak{R}, \bigcap_{\mathfrak{R} \in \mathfrak{I}_{1}} \mathfrak{R}$, and $\bigcap_{\mathfrak{R} \in \mathfrak{I}_{2}} \mathfrak{R}$ are called, respectively, proximal structure relation, $A \stackrel{(-)}{ }$ proximal structure relation (or simply $A$-proximal structure relation), $A \frac{(\overline{\mathrm{M}})}{\mathrm{p}}$ proximal structure relation, and $A \stackrel{(\overline{\overline{\mathrm{M}}})}{ }$ proximal structure relation (on $X$ ), for $a \in X$, instead of " $\{a\}$-proximal structure relation" we simply use " $a$-proximal structure relation"; and the sets

$$
\begin{gathered}
\mathrm{P}(X, S)=\{(x, y) \in X \times X \mid \exists I \in \operatorname{Min}(\mathrm{E}(X)) \forall p \in I x p=y p\} \\
\quad \text { (or simply } \mathrm{P}(X) \text { or } P), \\
\mathrm{P}_{A}(X, S)=\{(x, y) \in X \times X \mid \exists b \in A \exists I \in \mathrm{M}(b) \forall p \in I x p=y p\} \\
\text { (or simply } \left.\mathrm{P}_{A}(X) \text { or } \mathrm{P}_{A}\right), \\
\overline{\mathrm{P}}_{A}(X, S)=\{(x, y) \in X \times X \mid \exists I \in \overline{\mathrm{M}}(A) \forall p \in I x p=y p\} \\
\left.\quad \text { (or simply } \overline{\mathrm{P}}_{A}(X) \text { or } \overline{\mathrm{P}}_{A}\right), \\
\overline{\overline{\mathrm{P}}}_{A}(X, S)=\{(x, y) \in X \times X \mid \exists I \in \overline{\mathrm{M}}(A) \forall p \in I x p=y p\} \\
\left.\quad \text { (or simply } \overline{\overline{\mathrm{P}}}_{A}(X) \text { or } \overline{\overline{\mathrm{P}}}_{A}\right),
\end{gathered}
$$

are called, respectively, proximal relation, $A^{(-)}$proximal relation (or simply $A$-proximal relation), $A \stackrel{(\overline{\mathrm{M}})}{=}$ proximal relation, and $A \frac{(\overline{\overline{\mathrm{M}}})}{\mathrm{p}}$ proximal relation (on $\left.X\right)$, if $a \in X$, then instead of " $\{a\}$-proximal relation" (respectively, " $\mathrm{P}_{\{a\}}(X)$ ") we simply use " $a$-proximal relation" (respectively, " $\mathrm{P}_{a}(X)$ ").

TheOREM 1.2. Let $A$ be a nonempty subset of $X$, then by Definition 1.1, we have

(a) (i) if $\left\{\mathfrak{R}_{\alpha}\right\}_{\alpha \in \Gamma}$ is a nonempty collection in $\mathfrak{I}$, then $\bigcap_{\alpha \in \Gamma} \mathfrak{R}_{\alpha} \in \mathfrak{I}$,

(ii) if $\left\{\mathfrak{R}_{\alpha}\right\}_{\alpha \in \Gamma}$ is a nonempty collection in $\mathfrak{I}_{0}$, then $\bigcap_{\alpha \in \Gamma} \mathfrak{R}_{\alpha} \in \mathfrak{I}_{0}$,

(iii) if $\left\{\mathfrak{R}_{\alpha}\right\}_{\alpha \in \Gamma}$ is a nonempty collection in $\mathfrak{I}_{1}$ such that for $Z=\left\{\left(\left[x_{\alpha}\right]_{\mathfrak{R}_{\alpha}}\right)_{\alpha \in \Gamma} \mid\right.$ $x \in X\} \subseteq \prod_{\alpha \in \Gamma} X / \mathfrak{R}_{\alpha}$ we have $\left\{\left([a]_{\mathfrak{R}_{\alpha}}\right)_{\alpha \in \Gamma} \mid a \in A\right\} \in \overline{\mathcal{M}}(Z, S)$, then $\bigcap_{\alpha \in \Gamma}$ $\mathfrak{R}_{\alpha} \in \mathfrak{I}_{1}$,

(iv) if $\left\{\mathfrak{R}_{\alpha}\right\}_{\alpha \in \Gamma}$ is a nonempty collection in $\mathfrak{I}_{2}$ such that for $Z=\left\{\left(\left[x_{\alpha}\right]_{\mathfrak{R}_{\alpha}}\right)_{\alpha \in \Gamma} \mid\right.$ $x \in X\} \subseteq \prod_{\alpha \in \Gamma} X / \mathfrak{R}_{\alpha}$ we have $\left\{\left([a]_{\mathfrak{R}_{\alpha}}\right)_{\alpha \in \Gamma} \mid a \in A\right\} \in \overline{\bar{M}}(Z, S)$, then $\bigcap_{\alpha \in \Gamma}$ $\mathfrak{R}_{\alpha} \in \mathfrak{I}_{2}$,

(b) (i) $X \times X \in \mathfrak{I} \cap \mathfrak{J}_{0} \cap \mathfrak{I}_{1} \cap \mathfrak{J}_{2}$,

(ii) $\bigcap_{\mathfrak{R} \in \mathfrak{I}} \mathfrak{R} \in \mathfrak{J}, \bigcap_{\mathfrak{R} \in \mathfrak{I}_{0}} \mathfrak{R} \in \mathfrak{J}_{0}$,

(c) (i) $(X, S)$ is distal if and only if $\Delta_{X} \in \mathfrak{I}$,

(ii) $(X, S)$ is A-distal if and only if $\Delta_{X} \in \mathfrak{J}_{0}$,

(iii) $(X, S)$ is $A \frac{(\overline{\mathrm{M}})}{}$ distal if and only if $\Delta_{X} \in \mathfrak{I}_{1}$,

(iv) $(X, S)$ is $A \frac{(\overline{\overline{\mathrm{M}}})}{}$ distal if and only if $\Delta_{X} \in \mathfrak{I}_{2}$.

Proof. (a) (ii) Let $\left\{\mathfrak{R}_{\alpha}\right\}_{\alpha \in \Gamma}$ be a nonempty collection in $\mathfrak{J}_{0}$, then for each $\alpha \in \Gamma$, $\left(X / \mathfrak{R}_{\alpha}, S\right)$ is $[A]_{\mathfrak{R}_{\alpha}}$-distal, thus $\left(\prod_{\alpha \in \Gamma} X / \mathfrak{R}_{\alpha}, S\right)$ is $\left\{\left([a]_{\mathfrak{R}_{\alpha}}\right)_{\alpha \in \Gamma} \mid a \in A\right\}$-distal (since $\left.\left\{\left([a]_{\mathfrak{R}_{\alpha}}\right)_{\alpha \in \Gamma} \mid a \in A\right\} \subseteq \prod_{\alpha \in \Gamma}[A]_{\mathfrak{R}_{\alpha}}\right)$, but $\left\{\left([a]_{\mathfrak{R}_{\alpha}}\right)_{\alpha \in \Gamma} \mid a \in A\right\} \subseteq\left\{\left([x]_{\mathfrak{R}_{\alpha}}\right)_{\alpha \in \Gamma} \mid x \in\right.$ $X\}$ and $\left\{\left([x]_{\mathfrak{R}_{\alpha}}\right)_{\alpha \in \Gamma} \mid x \in X\right\}$ is a closed invariant subset of $\prod_{\alpha \in \Gamma} X / \mathfrak{R}_{\alpha}$, therefore $\left(\left\{\left([x]_{\mathfrak{R}_{\alpha}}\right)_{\alpha \in \Gamma} \mid x \in X\right\}, S\right)$ is $\left\{\left([a]_{\mathfrak{R}_{\alpha}}\right)_{\alpha \in \Gamma} \mid a \in A\right\}$-distal [4, Theorem 1.23(c)]. On the other hand, $\varphi:\left(X / \cap_{\alpha \in \Gamma} \mathfrak{R}_{\alpha}, S\right) \rightarrow\left(\left\{\left([x]_{\mathfrak{R}_{\alpha}}\right)_{\alpha \in \Gamma} \mid x \in X\right\}, S\right)$ defined by $\varphi\left([x]_{\cap_{\alpha \in \Gamma} \mathfrak{R}_{\alpha}}\right)=$ $\left([x]_{\mathfrak{K}_{\alpha}}\right)_{\alpha \in \Gamma}(\forall x \in X)$ is an isomorphism and $\left(\left\{\left([x]_{\mathfrak{R}_{\alpha}}\right)_{\alpha \in \Gamma} \mid x \in X\right\}, S\right)$ is 


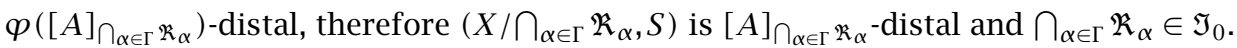

(iii) Let $\left\{\mathfrak{R}_{\alpha}\right\}_{\alpha \in \Gamma}$ be a nonempty collection in $\mathfrak{J}_{1}$, then for each $\alpha \in \Gamma,\left(X / \mathfrak{R}_{\alpha}, S\right)$ is $[A]_{\mathfrak{R}_{\alpha}} \frac{(\overline{\mathrm{M}})}{}$ distal, thus for each $\alpha \in \Gamma, J\left(F\left([A]_{\mathfrak{R}_{\alpha}}, \mathrm{E}\left(X / \mathfrak{R}_{\alpha}\right)\right)\right)=\{e\}$, therefore $J\left(F\left(\left\{\left([a]_{\mathfrak{R}_{\alpha}}\right)_{\alpha \in \Gamma} \mid a \in A\right\}, \mathrm{E}\left(\prod_{\alpha \in \Gamma} X / \mathfrak{R}_{\alpha}\right)\right)\right)=\{e\}$, but $\left\{\left([x]_{\mathfrak{R}_{\alpha}}\right)_{\alpha \in \Gamma} \mid x \in X\right\}$ is a closed invariant subset of $\prod_{\alpha \in \Gamma} X / \mathfrak{R}_{\alpha}$, and by the hypothesis $\left\{\left([a]_{\mathfrak{R}_{\alpha}}\right)_{\alpha \in \Gamma} \mid a \in A\right\} \in$ $\bar{M}\left(\left\{\left([x]_{\mathfrak{R}_{\alpha}}\right)_{\alpha \in \Gamma} \mid x \in X\right\}, S\right)$, thus $\left(\left\{\left([x]_{\mathfrak{R}_{\alpha}}\right)_{\alpha \in \Gamma} \mid x \in X\right\}, S\right)$ is $\left\{\left([a]_{\mathfrak{R}_{\alpha}}\right)_{\alpha \in \Gamma} \mid a \in A\right\} \stackrel{(\overline{\mathrm{M}})}{ }$ distal [4, Theorem 1.23(d)]. On the other hand, $\varphi:\left(X / \bigcap_{\alpha \in \Gamma} \mathfrak{R}_{\alpha}, S\right) \rightarrow\left(\left\{\left([x]_{\mathfrak{K}_{\alpha}}\right)_{\alpha \in \Gamma} \mid\right.\right.$ $x \in X\}, S)$ defined by $\varphi\left([x]_{\cap_{\alpha \in \Gamma} \mathfrak{R}_{\alpha}}\right)=\left([x]_{\mathfrak{R}_{\alpha}}\right)_{\alpha \in \Gamma}(\forall x \in X)$ is an isomorphism, and $\left(\left\{\left([x]_{\mathfrak{R}_{\alpha}}\right)_{\alpha \in \Gamma} \mid x \in X\right\}, S\right)$ is $\varphi\left([A]_{\text {cap }_{\alpha \in \Gamma} \mathfrak{R}_{\alpha}}\right) \frac{(\overline{\mathrm{M}})}{}$ distal, therefore $\left(X / \bigcap_{\alpha \in \Gamma} \mathfrak{R}_{\alpha}, S\right)$ is

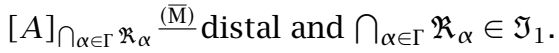

(iv) The proof is similar to (iii).

(b) Let $\mathfrak{R}=X \times X$, then $X / \mathfrak{R}$ is singleton, thus it is clear that $(X / \mathfrak{R}, S)$ is distal, $[A]_{\mathfrak{K}}$-distal, $[A]_{\mathfrak{R}} \frac{(\overline{\mathrm{M}})}{}$ distal, and $[A]_{\mathfrak{R}} \frac{(\overline{\overline{\mathrm{M}}})}{}$ distal, thus $X \times X=\mathfrak{R} \in \mathfrak{J} \cap \mathfrak{J}_{0} \cap \mathfrak{J}_{1} \cap \mathfrak{J}_{2}$. On the other hand, by (a) ((i) and (ii)) we have $\bigcap_{\mathfrak{R} \in \mathfrak{I}} \mathfrak{R} \in \mathfrak{I}$ and $\bigcap_{\mathfrak{R} \in \mathfrak{I}_{0}} \mathfrak{R} \in \mathfrak{I}_{0}$.

(c) (ii) Let $\mathfrak{R}=\Delta_{X}$, then the canonical map $\pi_{\mathfrak{R}}:(X, S) \rightarrow(X / \mathfrak{R}, S)$ is an isomorphism, thus $(X, S)$ is $A$-distal if and only if $(X / \mathfrak{R}, S)$ is $[A]_{\mathfrak{R}}$-distal if and only if $\Delta_{X}=\mathfrak{R} \in \mathfrak{I}_{0}$.

NoTE 1.3. Let $A$ be a nonempty subset of $X$, then

(a) (i) $\mathrm{P}(X)$ is a reflexive and symmetric relation on $X$,

(ii) $\mathrm{P}_{A}(X)$ is a reflexive and symmetric relation on $X$,

(iii) $\overline{\mathrm{P}}_{A}(X)$ is a reflexive and symmetric relation on $X$,

(iv) if $\overline{\overline{\mathrm{M}}}(A) \neq \varnothing$, then $\overline{\overline{\mathrm{P}}}_{A}(X)$ is a reflexive and symmetric relation on $X$,

(b) if $S$ is abelian, then $\mathrm{P}(X), \mathrm{P}_{A}(X), \overline{\mathrm{P}}_{A}(X)$, and $\overline{\overline{\mathrm{P}}}_{A}(X)$ (this latter case when $\overline{\overline{\mathrm{M}}}(A) \neq$

$\varnothing)$ are invariant relations on $X$,

(c) (i) for each nonempty subset $B$ of $A$ we have $\overline{\mathrm{P}}_{A}(X) \subseteq \overline{\mathrm{P}}_{B}(X) \subseteq \mathrm{P}_{B}(X) \subseteq \mathrm{P}_{A}(X) \subseteq$ $\mathrm{P}(X)$,

(ii) $\mathrm{P}_{A}(X)=\cup_{a \in A} \mathrm{P}_{a}(X)$,

(iii) $\overline{\mathrm{P}}_{A}(X) \subseteq \mathrm{P}(X)$,

(d) (i) if all of the points of $A$ are almost periodic, then $\overline{\overline{\mathrm{P}}}_{A}(X)=\overline{\mathrm{P}}_{A}(X)=\mathrm{P}_{A}(X)=$ $\mathrm{P}(X)$,

(ii) $\mathrm{P}_{A}(X)=\Delta_{X} \Leftrightarrow\left(\forall a \in A \mathrm{P}_{a}(X)=\Delta_{X}\right)$,

(iii) $\mathrm{P}_{A}(X)=\Delta_{X} \Rightarrow(\forall a \in A \forall K \in \mathrm{M}(a) J(F(a, K))=J(\mathrm{~S}(K))=\{e\})$,

(iv) $\overline{\mathrm{P}}_{A}(X)=\Delta_{X} \Rightarrow(\forall K \in \overline{\mathrm{M}}(A) J(F(A, K)) \subseteq J(\mathrm{~S}(K)) \subseteq\{e\})$,

(v) $\overline{\overline{\mathrm{P}}}_{A}(X)=\Delta_{X} \Rightarrow(\forall K \in \overline{\overline{\mathrm{M}}}(A) J(F(A, K)) \subseteq J(\mathrm{~S}(K)) \subseteq\{e\})$.

Proof. (a) and (b) are clear.

(c) (i) Let $B$ be a nonempty subset of $A$, then for each $(x, y) \in X \times X$ we have

$$
\begin{aligned}
(x, y) \in \overline{\mathrm{P}}_{A}(X) \\
\quad \Longrightarrow \exists K \in \overline{\mathrm{M}}(A) \forall p \in K, x p=y p \\
\quad \Longrightarrow \exists K \in \overline{\mathrm{M}}(A) \quad \exists L \in \overline{\mathrm{M}}(B) \forall p \in K, \quad(x p=y p \wedge L \subseteq K) \quad \text { by [4, Corollary 1.3]) } \\
\quad \Longrightarrow \exists L \in \overline{\mathrm{M}}(B) \forall p \in L, x p=y p \\
\quad \Longrightarrow(x, y) \in \overline{\mathrm{P}}_{B}(X)
\end{aligned}
$$


$a$-MINIMAL SETS AND RELATED TOPICS IN TRANSFORMATION ...

$$
\begin{aligned}
(x, y) \in \overline{\mathrm{P}}_{B}(X) & \\
\Longrightarrow & \exists L \in \overline{\mathrm{M}}(B) \forall p \in L, x p=y p \\
\Longrightarrow & \exists L \in \overline{\mathrm{M}}(B) \forall b \in B \exists K \in \mathrm{M}(b) \forall p \in L \\
& (x p=y p \wedge K \subseteq L) \quad(\text { by }[4, \text { Corollary 1.3]) } \\
\Longrightarrow & \forall b \in B \exists K \in \mathrm{M}(b) \forall p \in K, x p=y p \\
\Longrightarrow & \exists b \in B \exists K \in \mathrm{M}(b) \forall p \in K, x p=y p \\
\Longrightarrow & (x, y) \in \mathrm{P}_{B}(X) \\
(x, & y) \in \mathrm{P}_{B}(X) \\
\Longrightarrow & \exists b \in B \exists K \in \mathrm{M}(b) \forall p \in K, x p=y p \\
\Longrightarrow & \exists a \in A \exists K \in \mathrm{M}(a) \forall p \in K, x p=y p \\
\Longrightarrow & (x, y) \in \mathrm{P}_{A}(X) \\
(x, & y) \in \mathrm{P}_{A}(X) \\
\Longrightarrow & \exists a \in A \exists K \in \mathrm{M}(a) \forall p \in K, x p=y p \\
\Longrightarrow & \exists a \in A \exists K \in \mathrm{M}(a) \exists L \in \mathrm{Min}(\mathrm{E}(X)) \forall p \in K,(x p=y p \wedge L \subseteq K) \\
\Longrightarrow & \exists L \in \operatorname{Min}(\mathrm{E}(X)) \forall p \in L, x p=y p \\
\Longrightarrow & (x, y) \in \mathrm{P}(X) .
\end{aligned}
$$

(ii) It is clear by Definition 1.1.

(iii) It is clear by Definition 1.1 and the fact that each closed right ideal of $\mathrm{E}(X)$ contains at least one element of $\operatorname{Min}(\mathrm{E}(X))$.

(d) (i) If all of the points of $A$ are almost periodic, then for each $a \in A, \mathrm{M}(a)=$ $\overline{\mathrm{M}}(A)=\overline{\overline{\mathrm{M}}}(A)=\operatorname{Min}(\mathrm{E}(X))$ [4, Note 1.12], thus $\overline{\mathrm{P}}_{A}(X)=\overline{\mathrm{P}}_{A}(X)=\mathrm{P}_{A}(X)=\mathrm{P}(X)$.

(ii) Since for each $a \in A, \Delta_{X} \subseteq \mathrm{P}_{a}(X) \subseteq \mathrm{P}_{A}(X)=\cup_{b \in A} \mathrm{P}_{b}(X)$ (use (c) (ii)), thus $\mathrm{P}_{A}(X)=\Delta_{X}$ if and only if for each $a \in A, \mathrm{P}_{a}(X)=\Delta_{X}$.

(iv) Let $\overline{\mathrm{P}}_{A}(X)=\Delta_{X}$ and $K \in \overline{\mathrm{M}}(A)$, then $J(F(A, K)) \subseteq J(\mathrm{~S}(K))$ [4, Corollary 1.5(Table 1.3)], if $u \in J(\mathrm{~S}(K))$, then $u K=K$ and for each $x \in X,(x u) u=x u$, thus for each $p \in K,(x u) u p=x u p$, that is, for each $q \in K,(x u) q=x q$ and $(x u, x) \in \overline{\mathrm{P}}_{A}(X)=\Delta_{X}$, therefore for each $x \in X, x u=x$ and $u=e$.

Considering (ii), (iii) is a special case of (iv). The proof of (v) has a similar argument.

THEOREM 1.4. Let $A$ be a nonempty subset of $X$, then:

(a) (i) $(X, S)$ is distal if and only if $\mathrm{P}(X)=\Delta_{X}$,

(ii) $(X, S)$ is A-distal if and only if $\mathrm{P}_{A}(X)=\Delta_{X}$,

(iii) if $A \in \bar{M}(X, S)$, then $(X, S)$ is $A \frac{(\overline{\mathrm{M}})}{}$ distal if and only if $\overline{\mathrm{P}}_{A}(X)=\Delta_{X}$,

(iv) if $A \in \overline{\bar{M}}(X, S)$, then $(X, S)$ is $A \frac{(\overline{\bar{M}})}{2}$ distal if and only if $\overline{\overline{\mathrm{P}}}_{A}(X)=\Delta_{X}$,

(b) if $(x, y) \in X \times X$, then:

(i) the following statements are equivalent:

(1) $(x, y) \in \mathrm{P}(X)$,

(2) $\exists u \in J(\mathrm{E}(X)), x u=y u$,

(3) $\exists p \in \mathrm{E}(X), x p=y p$, 
(ii) the following statements are equivalent:

(1) $(x, y) \in \mathrm{P}_{A}(X)$,

(2) $\exists a \in A \exists u \in J(F(a, \mathrm{E}(X))), x u=y u$,

(3) $\exists a \in A \quad \exists p \in F(a, \mathrm{E}(X)), x p=y p$,

(iii) if $A \in \bar{M}(X, S)$, then the following statements are equivalent:

(1) $(x, y) \in \overline{\mathrm{P}}_{A}(X)$,

(2) $\exists u \in J(F(A, \mathrm{E}(X))), x u=y u$,

(3) $\exists p \in F(A, \mathrm{E}(X)), x p=y p$.

Proof. In each case for the sake of brevity we prove (iii).

(a) (iii) If $(X, S)$ if $A \frac{(\overline{\mathrm{M}})}{}$ distal, then $\overline{\mathrm{M}}(A)=\{\mathrm{E}(X)\}$, and if $(x, y) \in \overline{\mathrm{P}}_{A}(X)$, then for each $p \in \mathrm{E}(X), x p=y p$, thus $x=x e=y e=y$ and $\overline{\mathrm{P}}_{A}(X) \subseteq \Delta_{X}$, therefore $\overline{\mathrm{P}}_{A}(X)=$ $\Delta_{X}$. On the other hand, let $A \in \bar{M}(X, S)$ and $\overline{\mathrm{P}}_{A}(X)=\Delta_{X}$, take $K \in \overline{\mathrm{M}}(A)$ and $u \in$ $J(F(A, K))(\neq \varnothing)$, then $u K=K$ and for each $x \in X$ and $p \in K, x p=x(u p)=(x u) p$, so $(x, x u) \in \overline{\mathrm{P}}_{A}(X)=\Delta_{X}$, that is, for each $x \in X, x u=x$ and $u=e$ so $K=\mathrm{E}(X)$, therefore $(X, S)$ is $A \frac{(\overline{\mathrm{M}})}{}$ distal.

(b) (iii) We have

$$
\begin{aligned}
(1) & \Rightarrow \exists K \in \overline{\mathrm{M}}(A) \forall p \in K, x p=y p \\
\Longrightarrow & \exists K \in \overline{\mathrm{M}}(A) \exists u \in \mathrm{J}(\mathrm{F}(A, K)), x u=y u \text { (since } A \in \bar{M}(X, S)) \\
\Longrightarrow & (2), \\
(3) \Longrightarrow & \exists p \in \mathrm{F}(A, \mathrm{E}(X)) \forall q \in p \mathrm{E}(X), x q=y q \\
\Longrightarrow & \exists p \in \mathrm{F}(A, \mathrm{E}(X)) \exists L \in \overline{\mathrm{M}}(A) \forall q \in p \mathrm{E}(X), \\
& (x q=y q \wedge L \subseteq p \mathrm{E}(X)) \text { (by [4, Corollary 1.3]) } \\
\Longrightarrow & \exists L \in \overline{\mathrm{M}}(A) \forall q \in L, x q=y q \\
\Longrightarrow & (1) .
\end{aligned}
$$

THEOREM 1.5. Let A be a nonempty subset of $X$, then

(a) (i) the following statements are equivalent:

(1) $\operatorname{Min}(\mathrm{E}(X))$ is singleton,

(2) $\mathrm{P}(X)$ is a transitive relation on $X$,

(3) $\mathrm{P}(X)$ is an equivalence relation on $X$,

(ii) if $A \in \bar{M}(X, S)$, then the following statements are equivalent:

(1) $\overline{\mathrm{M}}(A)$ is singleton,

(2) $\overline{\mathrm{P}}_{A}(X)$ is a transitive relation on $X$,

(3) $\overline{\mathrm{P}}_{A}(X)$ is an equivalence relation on $X$,

(iii) if $A \in \overline{\bar{M}}(X, S)$, then the following statements are equivalent:

(1) $\overline{\overline{\mathrm{M}}}(A)$ is singleton,

(2) $\overline{\overline{\mathrm{P}}}_{A}(X)$ is a transitive relation on $X$,

(3) $\overline{\overline{\mathrm{P}}}_{A}(X)$ is an equivalence relation on $X$,

(b) if $S$ is an abelian semigroup, then:

(i) if $\mathrm{P}(X)$ is a closed relation on $X$, then $\mathrm{P}(X)$ is an equivalence relation on $X$,

(ii) if $A \in \bar{M}(X, S)$ and $\overline{\mathrm{P}}_{A}(X)$ is a closed relation on $X$, then $\overline{\mathrm{P}}_{A}(X)$ is an equivalence relation on $X$,

(iii) if $A \in \overline{\bar{M}}(X, S)$ and $\overline{\overline{\mathrm{P}}}_{A}(X)$ is a closed relation on $X$, then $\overline{\overline{\mathrm{P}}}_{A}(X)$ is an equivalence relation on $X$. 
Proof. (a) (ii) By Note 1.3(a), it is enough to show that (3) implies (1). Let $\overline{\mathrm{P}}_{A}(X)$ be an equivalence relation on $X, K, L \in \overline{\mathrm{M}}(A)$ and $u \in J(F(A, K))$, there exists $v \in$ $J(F(A, L))$ such that $u v=u$ and $v u=v$ [4, Theorem 7.1(a)], moreover, $u \mathrm{E}(X)=u K=$ $K, v \mathrm{E}(X)=v L=L[4$, Corollary 1.5(Table 3)], and for each $x \in X, p \in K$ and $q \in L$ we have: $(x u) p=x(u p)=x p$ and $(x v) q=x(v q)=x q$. Therefore $(x u, x),(x, x v) \in$ $\overline{\mathrm{P}}_{A}(X)$ and by the transitivity of $\overline{\mathrm{P}}_{A}(X),(x u, x v) \in \overline{\mathrm{P}}_{A}(X)$, thus there exists $N \in \overline{\mathrm{M}}(A)$ such that for each $l \in N, x u l=x v l$. We know there exists $w \in J(F(A, N))$, such that $u w=u$ and $v w=(v u) w=v(u w)=v u=v[4$, Theorem 1.7(a)] thus $x u=x u w=$ $x v w=x v$ (for each $x \in X$ ), so $u=v$ and $K=u \mathrm{E}(X)=v \mathrm{E}(X)=L$. Therefore $\overline{\mathrm{M}}(A)$ is singleton.

(b) (ii) Let $A \in \bar{M}(X, S)$ and $\overline{\mathrm{P}_{\mathrm{A}}}(X)$ be a closed relation on $X$, then for each $(x, y),(y, z)$ $\in \overline{\mathrm{P}}_{A}(X)$, there exists $K \in \overline{\mathrm{M}}(A)$ such that for each $p \in K, x p=y p$. Let $u \in J(F(A, K))$ $(\neq \varnothing)$, then $x u=y u$. Now by Note 1.3(b), we have $(y u, z u) \in \overline{\mathrm{P}}_{A}(X)$. Choose $L \in$ $\overline{\mathrm{M}}(A)$ such that for each $q \in L, y u q=z u q$. There exists $v \in J(F(A, L))$, such that $u v=u[4$, Theorem 1.7(a)] thus $x u=y u=y u v=z u v=z u$, by Theorem 1.4(iii), $(x, z) \in \overline{\mathrm{P}}_{A}(X)$ and $\overline{\mathrm{P}}_{A}(X)$ is a transitive relation on $X$, thus by (a (ii)) $\overline{\mathrm{P}}_{A}(X)$ is an equivalence relation on $X$.

Note 1.6. Let $A$ be a nonempty subset of $X$, let $\varphi:(X, S) \rightarrow(Y, S)$ be an onto homomorphism. Define $\varphi \times \varphi: X \times X \rightarrow Y \times Y$ by $\varphi \times \varphi(x, y)=(\varphi(x), \varphi(y))(\forall(x, y) \in$ $X \times X$ ), using Definition 1.1, we have

(a) if $K \in \overline{\mathrm{M}}(A)$, then there exists $L \in \overline{\mathrm{M}}(\varphi(A))$ such that $L \subseteq \hat{\varphi}(K)$,

(b) (i) $\varphi \times \varphi(\mathrm{P}(X)) \subseteq \mathrm{P}(Y)$,

(ii) $\varphi \times \varphi\left(\mathrm{P}_{A}(X)\right) \subseteq \mathrm{P}_{\varphi(A)}(Y)$,

(iii) $\varphi \times \varphi\left(\overline{\mathrm{P}}_{A}(X)\right) \subseteq \overline{\mathrm{P}}_{\varphi(A)}(Y)$,

(c) (i) $\mathrm{P}(X) \subseteq \bigcap_{\mathfrak{R} \in \mathfrak{I}} \mathfrak{R}$,

(ii) $\mathrm{P}_{A}(X) \subseteq \bigcap_{\mathfrak{R} \in \mathfrak{I}_{0}} \mathfrak{R}$,

(iii) $\overline{\mathrm{P}}_{A}(X) \subseteq \bigcap_{\mathfrak{R} \in \mathfrak{I}_{1}} \mathfrak{R}$.

Proof. (a) If $K \in \overline{\mathrm{M}}(A)$, then $\hat{\varphi}(K)$ is a closed right ideal of $\mathrm{E}(Y)$. On the other hand, for each $a \in A, a K=a \mathrm{E}(X)$ thus $\varphi(a) \hat{\varphi}(K)=\varphi(a K)=\varphi(a \mathrm{E}(X))=\varphi(a) \hat{\varphi}(\mathrm{E}(X))=$ $\varphi(a) \mathrm{E}(Y)$, therefore there exists $L \in \overline{\mathrm{M}}(\varphi(A))$ such that $L \subseteq \hat{\varphi}(K)$ [4, Corollary 1.3(b)].

(b) Let $(x, y) \in X \times X$.

(ii) If $(x, y) \in \mathrm{P}_{A}(X)$, then there exists $a \in A$ and $K \in \mathrm{M}(a)$ such that for each $p \in K$, $x p=y p$ and $\varphi(x) \hat{\varphi}(p)=\varphi(y) \hat{\varphi}(p)$, by (a) there exists $L \in \mathrm{M}(\varphi(a))$ such that $L \subseteq$ $\hat{\varphi}(K)$, so for each $q \in L, \varphi(x) q=\varphi(y) q$, therefore, $\varphi \times \varphi(x, y)=(\varphi(x), \varphi(y)) \in$ $\mathrm{P}_{\varphi(A)}(Y)$.

(iii) If $(x, y) \in \overline{\mathrm{P}}_{A}(X)$, then there exists $K \in \overline{\mathrm{M}}(A)$ such that for each $p \in K, x p=y p$ and $\varphi(x) \hat{\varphi}(p)=\varphi(y) \hat{\varphi}(p)$, by (a) there exists $L \in \overline{\mathrm{M}}(\varphi(A))$ such that $L \subseteq \hat{\varphi}(K)$, so for each $q \in L, \varphi(x) q=\varphi(y) q$, therefore, $\varphi \times \varphi(x, y)=(\varphi(x), \varphi(y)) \in \overline{\mathrm{P}}_{\varphi(A)}(Y)$.

(c) (ii) Let $\mathfrak{R} \in \mathfrak{I}_{0}$, then

$$
\begin{aligned}
\mathfrak{R} \in \mathfrak{I}_{0} & \Longrightarrow\left(\frac{X}{\mathfrak{R}}, S\right) \text { is }[A]_{\mathfrak{R}} \text {-distal } \\
& \Longrightarrow \mathrm{P}_{[A]_{\mathfrak{R}}}\left(\frac{X}{\mathfrak{R}}\right)=\Delta_{X / \mathfrak{R}}
\end{aligned}
$$




$$
\begin{aligned}
& \Longrightarrow \pi_{\mathfrak{R}} \times \pi_{\mathfrak{R}}\left(\mathrm{P}_{A}(X)\right) \subseteq \mathrm{P}_{\pi_{\mathfrak{R}}(A)}\left(\frac{X}{\mathfrak{R}}\right)=\mathrm{P}_{[A]_{\mathfrak{R}}}\left(\frac{X}{\mathfrak{R}}\right)=\Delta_{X / \mathfrak{R}} \\
& \Longrightarrow \pi_{\mathfrak{R}} \times \pi_{\mathfrak{R}}\left(\mathrm{P}_{A}(X)\right)=\Delta_{X / \mathfrak{R}} \\
& \Longrightarrow \forall(x, y) \in \mathrm{P}_{A}(X),[x]_{\mathfrak{R}}=[y]_{\mathfrak{R}} \\
& \Longrightarrow \forall(x, y) \in \mathrm{P}_{A}(X),(x, y) \in \mathfrak{R} \\
& \Longrightarrow \mathrm{P}_{A}(X) \subseteq \mathfrak{R}
\end{aligned}
$$

so $\mathrm{P}_{A}(X) \subseteq \bigcap_{\mathfrak{R} \in \mathfrak{I}_{0}} \mathfrak{R}$.

DeFinITION 1.7. Let $\varphi:(X, S) \rightarrow(Y, S)$ be an onto homomorphism, $\mathrm{R}(\varphi)=$ $\{(x, y) \in X \times X \mid \varphi(x)=\varphi(y)\}$, and let $A$ be a nonempty subset of $X$, and let $B$ be a nonempty subset of $Y$, then

(a) $(Y, S)$ is a distal factor of $(X, S)$ (under $\varphi$ ) if $\mathrm{R}(\varphi) \cap \mathrm{P}(X)=\Delta_{X}$,

(b) $(Y, S)$ is an $A \frac{(-)}{}$ distal (or simply $A$-distal) factor of $(X, S)$ (under $\varphi$ ) if $\mathrm{R}(\varphi) \cap$ $\mathrm{P}_{A}(X)=\Delta_{X}$

(c) $(Y, S)$ is an $A \frac{(\overline{\mathrm{M}})}{}$ distal factor of $(X, S)$ (under $\varphi$ ) if $\mathrm{R}(\varphi) \cap \overline{\mathrm{P}}_{A}(X)=\Delta_{X}$,

(d) $(Y, S)$ is an $A \frac{(\overline{\overline{\mathrm{M}}})}{}$ distal factor of $(X, S)$ (under $\left.\varphi\right)$ if $\mathrm{R}(\varphi) \cap \overline{\overline{\mathrm{P}}}_{A}(X)=\Delta_{X}$,

(e) $(X, S)$ is a distal extension of $(Y, S)$ (under $\varphi)$ if $\mathrm{R}(\varphi) \cap \mathrm{P}(X)=\Delta_{X}$,

(f) $(X, S)$ is a $B \frac{(-)}{-}$ distal (or simply $B$-distal) extension of $(Y, S)$ (under $\varphi$ ) if $\mathrm{R}(\varphi) \cap$ $\mathrm{P}_{\varphi^{-1}(B)}(X)=\Delta_{X}$,

(g) $(X, S)$ is a $B \frac{(\overline{\mathrm{M}})}{}$ distal extension of $(Y, S)$ (under $\varphi$ ) if $\mathrm{R}(\varphi) \cap \overline{\mathrm{P}}_{\varphi^{-1}(B)}(X)=\Delta_{X}$,

(h) $(X, S)$ is a $B \frac{(\overline{\bar{M}})}{}$ distal extension of $(Y, S)$ (under $\varphi$ ) if $\mathrm{R}(\varphi) \cap \overline{\overline{\mathrm{P}}}_{\varphi^{-1}(B)}(X)=\Delta_{X}$,

(a) $^{\prime} \quad(Y, S)$ is a proximal factor of $(X, S)$ (under $\varphi$ ) if $\mathrm{R}(\varphi) \subseteq \mathrm{P}(X)$,

(b) $(Y, S)$ is an $A \stackrel{(-)}{-}$ proximal (or simply $A$-proximal) factor of $(X, S)$ (under $\varphi$ ) if $\mathrm{R}(\varphi) \subseteq \mathrm{P}_{A}(X)$,

(c) $(Y, S)$ is an $A \stackrel{(\overline{\mathrm{M}})}{-}$ proximal factor of $(X, S)$ (under $\varphi$ ) if $\mathrm{R}(\varphi) \subseteq \overline{\mathrm{P}}_{A}(X)$,

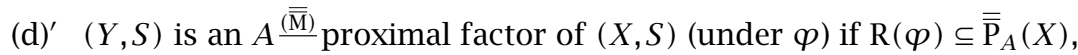

(e) $(X, S)$ is a proximal extension of $(Y, S)$ (under $\varphi$ ) if $\mathrm{R}(\varphi) \subseteq \mathrm{P}(X)$,

(f) $(X, S)$ is a $B \frac{(-)}{}$ proximal (or simply $B$-proximal) extension of $(Y, S)$ (under $\left.\varphi\right)$ if $\mathrm{R}(\varphi) \subseteq \mathrm{P}_{\varphi^{-1}(B)}(X)$

(g) $(X, S)$ is a $B \frac{(\overline{\mathrm{M}})}{}$ proximal extension of $(Y, S)$ (under $\left.\varphi\right)$ if $\mathrm{R}(\varphi) \subseteq \overline{\mathrm{P}}_{\varphi^{-1}(B)}(X)$,

$(\mathrm{h})^{\prime}(X, S)$ is a $B \frac{(\overline{\overline{\mathrm{M}}})}{}$ proximal extension of $(Y, S)$ (under $\left.\varphi\right)$ if $\mathrm{R}(\varphi) \subseteq \overline{\overline{\mathrm{P}}}_{\varphi^{-1}(B)}(X)$.

THEOREM 1.8. Let $\varphi:(X, S) \rightarrow(Y, S)$ be an onto homomorphism, let $A$ be a nonempty subset of $X$, let $B$ be a nonempty subset of $Y$, and consider the following statements:

$\left(\pi_{1}\right)(Y, S)$ is a distal factor of $(X, S)$ under $\varphi$,

$\left(\pi_{2}\right)(Y, S)$ is an A-distal factor of $(X, S)$ under $\varphi$,

$\left(\pi_{3}\right)(Y, S)$ is an $A \frac{(\overline{\mathrm{M}})}{}$ distal factor of $(X, S)$ under $\varphi$,

$\left(\pi_{4}\right)(Y, S)$ is an $A \frac{(\overline{\overline{\mathrm{M}}})}{}$ distal factor of $(X, S)$ under $\varphi$ (by the assumption $\overline{\overline{\mathrm{M}}}(A) \neq \varnothing$ ),

$\left(\rho_{1}\right) \quad(X, S)$ is a distal extension of $(Y, S)$ under $\varphi$,

$\left(\rho_{2}\right) \quad(X, S)$ is a $B$-distal extension of $(Y, S)$ under $\varphi$,

$\left(\rho_{3}\right)(X, S)$ is a $B \frac{(\overline{\mathrm{M}})}{}$ distal extension of $(Y, S)$ under $\varphi$,

$\left(\rho_{4}\right) \quad(X, S)$ is a $B \frac{(\overline{\bar{M}})}{}$ distal extension of $(Y, S)$ under $\varphi$ (by the assumption $\overline{\overline{\mathrm{M}}}\left(\varphi^{-1}(B)\right.$ ) $\neq \varnothing)$, 
$\left(\pi_{1}^{\prime}\right)(Y, S)$ is a proximal factor of $(X, S)$ under $\varphi$,

$\left(\pi_{2}^{\prime}\right)(Y, S)$ is an A-proximal factor of $(X, S)$ under $\varphi$,

$\left(\pi_{3}^{\prime}\right) \quad(Y, S)$ is an $A \frac{(\overline{\mathrm{M}})}{\mathrm{p}}$ proximal factor of $(X, S)$ under $\varphi$,

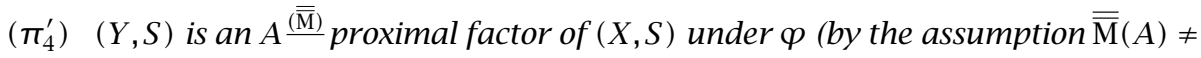
$\varnothing)$,

$\left(\rho_{1}^{\prime}\right)(X, S)$ is a proximal extension of $(Y, S)$ under $\varphi$,

$\left(\rho_{2}^{\prime}\right) \quad(X, S)$ is a B-proximal extension of $(Y, S)$ under $\varphi$,

$\left(\rho_{3}^{\prime}\right) \quad(X, S)$ is a $B \frac{(\overline{\mathrm{M}})}{2}$ proximal extension of $(Y, S)$ under $\varphi$,

$\left(\rho_{4}^{\prime}\right) \quad(X, S)$ is a $B \frac{(\overline{\bar{M}})}{2}$ proximal extension of $(Y, S)$ under $\varphi$ (by the assumption $\left.\overline{\overline{\mathrm{M}}}\left(\varphi^{-1}(B)\right) \neq \varnothing\right)$,

then we have the following tables:

TABLE 1.1. The mark " $\sqrt{ }$ " indicates that for the corresponding case we have:

“ $\left(\pi_{i} \Rightarrow \pi_{j}\right) \wedge\left(\rho_{i} \Rightarrow \rho_{j}\right)$ ”

\begin{tabular}{c|cccc}
\hline$j$ & \multirow{2}{*}{1} & 2 & 3 & 4 \\
\cline { 1 - 4 }$i$ & & & & \\
\hline 1 & $\sqrt{ }$ & $\sqrt{ }$ & $\sqrt{ }$ & $\sqrt{ }$ \\
2 & & $\sqrt{ }$ & $\sqrt{ }$ & \\
3 & & & $\sqrt{ }$ & \\
4 & & & & $\sqrt{ }$ \\
\hline
\end{tabular}

TABLE 1.2. The mark " $\sqrt{ }$ " indicates that for the corresponding case we have: “( $\left(\pi_{i}^{\prime} \Rightarrow \pi_{j}^{\prime}\right) \wedge\left(\rho_{i}^{\prime} \Rightarrow \rho_{j}^{\prime}\right) ”$

\begin{tabular}{l|llll}
\hline$j$ & 1 & 2 & 3 & 4 \\
\hline$i$ & & & & \\
\hline 1 & $\sqrt{ }$ & & & \\
2 & $\sqrt{ }$ & $\sqrt{ }$ & & \\
3 & $\sqrt{ }$ & $\sqrt{ }$ & $\sqrt{ }$ & \\
4 & $\sqrt{ }$ & & & $\sqrt{ }$ \\
\hline
\end{tabular}

Proof. We have the following conditional statements:

$$
\begin{aligned}
\left(\pi_{1}\right) \Longrightarrow & \mathrm{R}(\varphi) \cap \mathrm{P}(X)=\Delta_{X} \\
\Longrightarrow & \left(\mathrm{R}(\varphi) \cap \overline{\mathrm{P}}_{A}(X) \subseteq \mathrm{R}(\varphi) \cap \mathrm{P}_{A}(X) \subseteq \mathrm{R}(\varphi) \cap \mathrm{P}(X)=\Delta_{X}\right. \\
& \left.\wedge \mathrm{R}(\varphi) \cap \overline{\overline{\mathrm{P}}}_{A}(X) \subseteq \mathrm{R}(\varphi) \cap \mathrm{P}(X)=\Delta_{X}\right) \text { (by Note 1.3(c)) } \\
\Longrightarrow & \left(\mathrm{R}(\varphi) \cap \overline{\mathrm{P}}_{A}(X)=\mathrm{R}(\varphi) \cap \mathrm{P}_{A}(X)=\Delta_{X} \wedge \mathrm{R}(\varphi) \cap \overline{\overline{\mathrm{P}}}_{A}(X) \subseteq \Delta_{X}\right) \\
\Longrightarrow & \left(\pi_{2} \wedge \pi_{3} \wedge \pi_{4}\right), \\
\left(\rho_{1}\right) \Longrightarrow & \mathrm{R}(\varphi) \cap \mathrm{P}(X)=\Delta_{X} \\
\Longrightarrow & \left(\mathrm{R}(\varphi) \cap \overline{\mathrm{P}}_{\varphi^{-1}(B)}(X) \subseteq \mathrm{R}(\varphi) \cap \mathrm{P}_{\varphi^{-1}(B)}(X) \subseteq \mathrm{R}(\varphi) \cap \mathrm{P}(X)=\Delta_{X}\right. \\
& \left.\wedge \mathrm{R}(\varphi) \cap \overline{\overline{\mathrm{P}}}_{\varphi^{-1}(B)}(X) \subseteq \mathrm{R}(\varphi) \cap \mathrm{P}(X)=\Delta_{X}\right)(\text { by Note 1.3(c)) } \\
\Longrightarrow & \left(\mathrm{R}(\varphi) \cap \overline{\mathrm{P}}_{\varphi^{-1}(B)}(X)=\mathrm{R}(\varphi) \cap \mathrm{P}_{\varphi^{-1}(B)}(X)=\Delta_{X}\right. \\
& \left.\wedge \mathrm{R}(\varphi) \cap \overline{\overline{\mathrm{P}}}_{\varphi^{-1}(B)}(X) \subseteq \Delta_{X}\right)
\end{aligned}
$$




$$
\begin{aligned}
& \Longrightarrow\left(\rho_{2} \wedge \rho_{3} \wedge \rho_{4}\right), \\
\left(\pi_{2}\right) & \Longrightarrow \mathrm{R}(\varphi) \cap \mathrm{P}_{A}(X)=\Delta_{X} \\
& \Longrightarrow \mathrm{R}(\varphi) \cap \overline{\mathrm{P}}_{A}(X) \subseteq \mathrm{R}(\varphi) \cap \mathrm{P}_{A}(X)=\Delta_{X} \text { (by Note 1.3(c)) } \\
& \Longrightarrow \mathrm{R}(\varphi) \cap \overline{\mathrm{P}}_{A}(X)=\Delta_{X} \\
& \Longrightarrow\left(\pi_{3}\right), \\
\left(\rho_{2}\right) & \Longrightarrow \mathrm{R}(\varphi) \cap \mathrm{P}_{\varphi^{-1}(B)}(X)=\Delta_{X} \\
& \left.\Longrightarrow \mathrm{R}(\varphi) \cap \overline{\mathrm{P}}_{\varphi^{-1}(B)}(X) \subseteq \mathrm{R}(\varphi) \cap \mathrm{P}_{\varphi^{-1}(B)}(X)=\Delta_{X} \text { (by Note } 1.3(\mathrm{c})\right) \\
& \Longrightarrow \mathrm{R}(\varphi) \cap \overline{\mathrm{P}}_{\varphi^{-1}(B)}(X)=\Delta_{X} \\
& \Longrightarrow\left(\rho_{3}\right)
\end{aligned}
$$

these complete the proof of Table 1.1, also

$$
\begin{aligned}
\left(\pi_{3}^{\prime}\right) & \Longrightarrow \mathrm{R}(\varphi) \subseteq \overline{\mathrm{P}}_{A}(X) \\
& \left.\Longrightarrow \mathrm{R}(\varphi) \subseteq \overline{\mathrm{P}}_{A}(X) \subseteq \mathrm{P}_{A}(X) \text { (by Note } 1.3(\mathrm{c})\right) \\
& \Longrightarrow\left(\pi_{2}^{\prime}\right) \\
& \left.\Longrightarrow \mathrm{R}(\varphi) \subseteq \mathrm{P}_{A}(X) \subseteq \mathrm{P}(X) \text { (by Note } 1.3(\mathrm{c})\right) \\
& \Longrightarrow\left(\pi_{1}^{\prime}\right), \\
\left(\rho_{3}^{\prime}\right) & \Longrightarrow \mathrm{R}(\varphi) \subseteq \overline{\mathrm{P}}_{\varphi^{-1}(B)}(X) \\
& \left.\Longrightarrow \mathrm{R}(\varphi) \subseteq \overline{\mathrm{P}}_{\varphi^{-1}(B)}(X) \subseteq \mathrm{P}_{\varphi^{-1}(B)}(X) \text { (by Note } 1.3(\mathrm{c})\right) \\
& \Longrightarrow\left(\rho_{2}^{\prime}\right) \\
& \left.\Longrightarrow \mathrm{R}(\varphi) \subseteq \mathrm{P}_{\varphi^{-1}(B)}(X) \subseteq \mathrm{P}(X) \text { (by Note } 1.3(\mathrm{c})\right) \\
& \Longrightarrow\left(\rho_{1}^{\prime}\right), \\
\left(\pi_{4}^{\prime}\right) & \Longrightarrow \mathrm{R}(\varphi) \subseteq \overline{\mathrm{P}}_{A}(X) \\
& \Longrightarrow \mathrm{R}(\varphi) \subseteq \overline{\mathrm{P}}_{A}(X) \subseteq \mathrm{P}(X)(\text { by Note } 1.3(\mathrm{c})) \\
& \Longrightarrow\left(\pi_{1}^{\prime}\right), \\
\left(\rho_{4}^{\prime}\right) & \Longrightarrow \mathrm{R}(\varphi) \subseteq \overline{\overline{\mathrm{P}}}_{\varphi^{-1}(B)}(X) \\
& \Longrightarrow \mathrm{R}(\varphi) \subseteq \overline{\overline{\mathrm{P}}}_{\varphi^{-1}(B)}(X) \subseteq \mathrm{P}(X) \text { (by Note 1.3(c))} \\
& \Longrightarrow\left(\rho_{1}^{\prime}\right),
\end{aligned}
$$

these complete the proof of Table 1.2.

THEOREM 1.9. Let $\varphi:(X, S) \rightarrow(Y, S)$ be an onto homomorphism and $\varnothing \neq C \subseteq A \subseteq X$, and $\varnothing \neq D \subseteq B \subseteq Y$, then

(a) if $(Y, S)$ is an A-distal factor of $(X, S)$, then $(Y, S)$ is a $C$-distal factor of $(X, S)$,

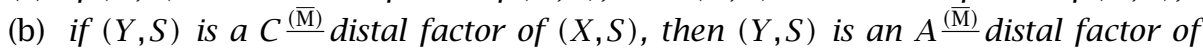
$(X, S)$,

(c) if $(X, S)$ is a B-distal extension of $(Y, S)$, then $(X, S)$ is a $D$-distal extension of $(Y, S)$,

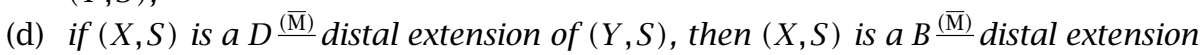
of $(Y, S)$, 
(e) if $(Y, S)$ is a C-proximal factor of $(X, S)$, then $(Y, S)$ is an A-proximal factor of $(X, S)$,

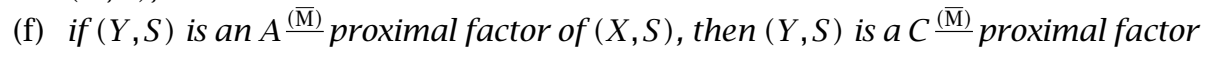
of $(X, S)$

(g) if $(X, S)$ is a D-proximal extension of $(Y, S)$, then $(X, S)$ is a B-proximal extension of $(Y, S)$,

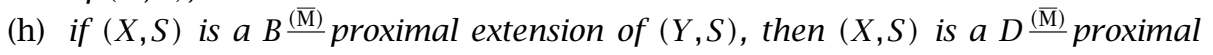
extension of $(Y, S)$,

the factors and extensions are under $\varphi$.

Proof. (a) $(Y, S)$ is an $A$-distal factor of $(X, S)$

$$
\begin{aligned}
& \Longrightarrow \mathrm{R}(\varphi) \cap \mathrm{P}_{A}(X)=\Delta_{X} \\
& \Longrightarrow \mathrm{R}(\varphi) \cap \mathrm{P}_{C}(X) \subseteq \mathrm{R}(\varphi) \cap \mathrm{P}_{A}(X)=\Delta_{X} \text { (by Note 1.3(c)) } \\
& \Longrightarrow \mathrm{R}(\varphi) \cap \mathrm{P}_{C}(X)=\Delta_{X} \\
& \Longrightarrow(Y, S) \text { is a } C \text {-distal factor of }(X, S),
\end{aligned}
$$

(b) $(Y, S)$ is a $C \stackrel{(\overline{\mathrm{M}})}{ }$ distal factor of $(X, S)$

$$
\begin{aligned}
& \Longrightarrow \mathrm{R}(\varphi) \cap \overline{\mathrm{P}}_{C}(X)=\Delta_{X} \\
& \Longrightarrow \mathrm{R}(\varphi) \cap \overline{\mathrm{P}}_{A}(X) \subseteq \mathrm{R}(\varphi) \cap \overline{\mathrm{P}}_{C}(X)=\Delta_{X} \text { (by Note 1.3(c)) } \\
& \Rightarrow \mathrm{R}(\varphi) \cap \overline{\mathrm{P}}_{A}(X)=\Delta_{X} \\
& \Longrightarrow(Y, S) \text { is an } A \frac{(\overline{\mathrm{M}})}{} \text { distal factor of }(X, S)
\end{aligned}
$$

(e) $(Y, S)$ is a $C$-proximal factor of $(X, S)$

$$
\begin{aligned}
& \Longrightarrow \mathrm{R}(\varphi) \subseteq \mathrm{P}_{C}(X) \\
& \left.\Longrightarrow \mathrm{R}(\varphi) \subseteq \mathrm{P}_{C}(X) \subseteq \mathrm{P}_{A}(X) \text { (by Note } 1.3(\mathrm{c})\right) \\
& \Longrightarrow(Y, S) \text { is an } A \text {-proximal factor of }(X, S),
\end{aligned}
$$

(f) $(Y, S)$ is an $A \stackrel{(\overline{\mathrm{M}})}{ }$ proximal factor of $(X, S)$

$$
\begin{aligned}
& \Longrightarrow \mathrm{R}(\varphi) \subseteq \overline{\mathrm{P}}_{A}(X) \\
& \left.\Longrightarrow \mathrm{R}(\varphi) \subseteq \overline{\mathrm{P}}_{A}(X) \subseteq \overline{\mathrm{P}}_{C}(X) \text { (by Note } 1.3(\mathrm{c})\right) \\
& \Longrightarrow(Y, S) \text { is a } C \frac{(\overline{\mathrm{M}})}{} \text { proximal factor of }(X, S) .
\end{aligned}
$$

Theorem 1.10 (associative and inheritance laws). Let $\varphi:(X, S) \rightarrow(Y, S)$ and $\psi:$ $(Y, S) \rightarrow(Z, S)$ be two onto homomorphisms, and let $A$ be a nonempty subset of $X$, and $B$ be a nonempty subset of $Y$, then we have

\section{(a) Associative LAws.}

(i) $(((Z, S)$ is a distal factor of $(Y, S)$ (under $\psi)) \wedge((Y, S)$ is a distal factor of $(X, S)$ (under $\varphi))) \Rightarrow((Z, S)$ is a distal factor of $(X, S)$ (under $\psi \circ \varphi))$,

(ii) $(((Z, S)$ is a $\varphi(A)$-distal factor of $(Y, S)$ (under $\psi)) \wedge((Y, S)$ is an A-distal factor of $(X, S)($ under $\varphi))) \Rightarrow((Z, S)$ is an A-distal factor of $(X, S)($ under $\psi \circ \varphi))$, 


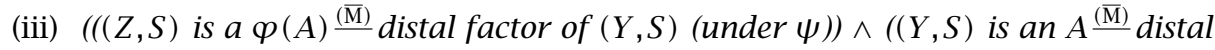

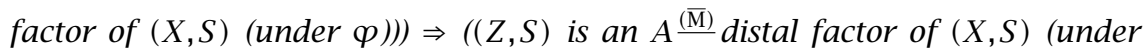
$\psi \circ \varphi))$,

(i) $\quad(((X, S)$ is a distal extension of $(Y, S)$ (under $\varphi)) \wedge((Y, S)$ is a distal extension of $(Z, S)($ under $\psi))) \Rightarrow((X, S)$ is a distal extension of $(Z, S)($ under $\psi \circ \varphi))$,

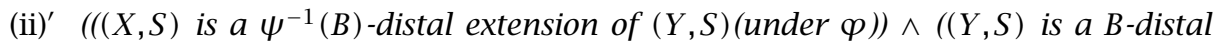
extension of $(Z, S)$ (under $\psi))) \Rightarrow((X, S)$ is a $B$-distal extension of $(Z, S)$ (under $\psi \circ \varphi))$,

(iii) $^{\prime} \quad\left(\left((X, S)\right.\right.$ is $a \psi^{-1}(B) \frac{(\overline{\mathrm{M}})}{2}$ distal extension of $(Y, S)($ under $\left.\varphi)\right) \wedge\left((Y, S)\right.$ is a $B \frac{(\overline{\mathrm{M}})}{\text { distal }}$ extension of $(Z, S)($ under $\psi))) \Rightarrow\left((X, S)\right.$ is a $B \frac{(\overline{\mathrm{M}})}{}$ distal extension of $(Z, S)$ (under $\psi \circ \varphi))$,

(b) INHERITANCE LAWS.

(i) $((Z, S)$ is a distal factor of $(X, S)$ (under $\psi \circ \varphi)) \Rightarrow((Y, S)$ is a distal factor of $(X, S)$ (under $\varphi)$ ),

(ii) $((Z, S)$ is an A-distal factor of $(X, S)($ under $\psi \circ \varphi)) \Rightarrow((Y, S)$ is an A-distal factor of $(X, S)$ (under $\varphi)$ ),

(iii) $\left((Z, S)\right.$ is an $A \frac{(\overline{\mathrm{M}})}{}$ distal factor of $(X, S)$ (under $\left.\left.\psi \circ \varphi\right)\right) \Rightarrow\left((Y, S)\right.$ is an $A^{(\overline{\mathrm{M}})}$ distal factor of $(X, S)$ (under $\varphi)$ ),

(iv) $\left((Z, S)\right.$ is an $A \frac{(\overline{\overline{\mathrm{M}}})}{}$ distal factor of $(X, S)($ under $\left.\psi \circ \varphi)\right) \Rightarrow\left((Y, S)\right.$ is an $A \frac{(\overline{\overline{\mathrm{M}}})}{}$ distal factor of $(X, S)$ (under $\varphi)$ ),

(v) $((Z, S)$ is a proximal factor of $(X, S)$ (under $\psi \circ \varphi)) \Rightarrow((Y, S)$ is a proximal factor of $(X, S)$ (under $\varphi)$ ),

(vi) $((Z, S)$ is an A-proximal factor of $(X, S)$ (under $\psi \circ \varphi)) \Rightarrow((Y, S)$ is an A-proximal factor of $(X, S)$ (under $\varphi)$ ),

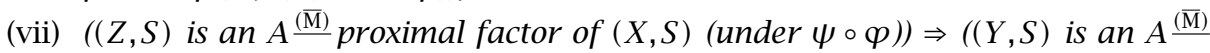
proximal factor of $(X, S)$ (under $\varphi)$ ),

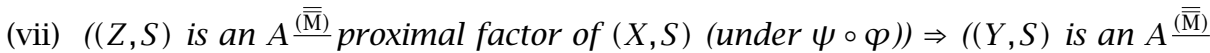
proximal factor of $(X, S)$ (under $\varphi)$ ),

(i) $\quad((X, S)$ is a distal extension of $(Z, S)($ under $\psi \circ \varphi)) \Rightarrow((X, S)$ is a distal extension of $(Y, S)$ (under $\varphi)$ ),

(ii) $^{\prime} \quad((X, S)$ is a B-distal extension of $(Z, S)($ under $\psi \circ \varphi)) \Rightarrow\left((X, S)\right.$ is a $\psi^{-1}(B)$-distal extension of $(Y, S)$ (under $\varphi)$ ),

(iii) $^{\prime} \quad\left((X, S)\right.$ is a $B \frac{(\bar{M})}{}$ distal extension of $(Z, S)($ under $\left.\psi \circ \varphi)\right) \Rightarrow\left((X, S)\right.$ is a $\psi^{-1}(B) \frac{(\bar{M})}{}$ distal extension of $(Y, S)$ (under $\varphi)$ ),

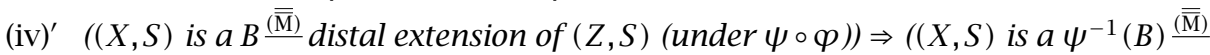
distal extension of $(Y, S)$ (under $\varphi)$ ),

$(\mathrm{v})^{\prime} \quad((X, S)$ is a proximal extension of $(Z, S)$ (under $\left.\psi \circ \varphi)\right) \Rightarrow((X, S)$ is a proximal extension of $(Y, S)$ (under $\varphi)$ ),

$(\mathrm{vi})^{\prime} \quad((X, S)$ is a B-proximal extension of $(Z, S)$ (under $\left.\psi \circ \varphi)\right) \Rightarrow\left((X, S)\right.$ is a $\psi^{-1}(B)$ proximal extension of $(Y, S)$ (under $\varphi)$ ),

(vii) $^{\prime}\left((X, S)\right.$ is a $B \frac{(\overline{\mathrm{M}})}{}$ proximal extension of $(Z, S)$ (under $\left.\left.\psi \circ \varphi\right)\right) \Rightarrow((X, S)$ is a $\psi^{-1}(B) \stackrel{(\bar{M})}{=}$ proximal extension of $(Y, S)$ (under $\left.\varphi\right)$ ), 


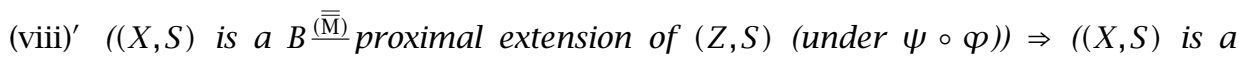
$\psi^{-1}(B) \stackrel{(\overline{\bar{M}})}{~ p r o x i m a l ~ e x t e n s i o n ~ o f ~}(Y, S)($ under $\left.\varphi)\right)$.

Proof. (a) (ii) Let $(Z, S)$ be a $\varphi(A)$-distal factor of $(Y, S)$ under $\psi$, and let $(Y, S)$ be an $A$-distal factor of $(X, S)$ under $\varphi$, then $\mathrm{R}(\psi) \cap \mathrm{P}_{\varphi(A)}(Y)=\Delta_{Y}$ and $\mathrm{R}(\varphi) \cap \mathrm{P}_{A}(X)=$ $\Delta_{X}$. Moreover, using the symbols of Note 1.6, we have $\varphi \times \varphi(\mathrm{R}(\psi \circ \varphi)) \subseteq \mathrm{R}(\psi)$ so $\varphi \times \varphi\left(\mathrm{R}(\psi \circ \varphi) \cap \mathrm{P}_{A}(X)\right) \subseteq \mathrm{R}(\psi) \cap \mathrm{P}_{\varphi(A)}(Y)=\Delta_{Y}$, thus $\varphi \times \varphi\left(\mathrm{R}(\psi \circ \varphi) \cap \mathrm{P}_{A}(X)\right)=\Delta_{Y}$, that is, $\mathrm{R}(\psi \circ \varphi) \cap \mathrm{P}_{A}(X) \subseteq \mathrm{R}(\varphi)$, thus $\mathrm{R}(\psi \circ \varphi) \cap \mathrm{P}_{A}(X) \subseteq \mathrm{R}(\varphi) \cap \mathrm{P}_{A}(X)$, therefore $\mathrm{R}(\psi \circ \varphi) \cap \mathrm{P}_{A}(X)=\Delta_{X}$ and $(Z, S)$ is an $A$-distal factor of $(X, S)$ (under $\psi \circ \varphi$ ).

(b) Use $\mathrm{R}(\varphi) \subseteq \mathrm{R}(\psi \circ \varphi)$.

THEOREM 1.11. Let $B$ be a nonempty subset of $X$, let $\Sigma=\left\{\varphi_{\alpha} \mid \alpha \in \Gamma\right\}$ be a nonempty collection of the extensions of $(X, S), \alpha_{0} \in \Gamma, \times_{\Sigma} X_{\alpha}=\left\{\left(x_{\alpha}\right)_{\alpha \in \Gamma} \in \prod_{\alpha \in \Gamma} X_{\alpha} \mid \forall \alpha \in\right.$ $\left.\Gamma \varphi_{\alpha}\left(x_{\alpha}\right)=\varphi_{\alpha_{0}}\left(x_{\alpha_{0}}\right)\right\}$, for each $\gamma \in \Gamma$ let $\pi_{\gamma}: \times_{\Sigma} X_{\alpha} \rightarrow X_{\gamma}$ be the projection map on the $\gamma$ th coordinate, and $\varphi: \times_{\Sigma} X_{\alpha} \rightarrow X$ be such that $\varphi\left(\left(x_{\alpha}\right)_{\alpha \in \Gamma}\right)=\varphi_{\alpha_{0}}\left(x_{\alpha_{0}}\right)$, then

(a) for each $\gamma \in \Gamma$, the following diagram commutes:

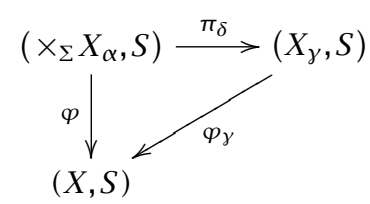

(b) (i) if for each $\alpha \in \Gamma,\left(X_{\alpha}, S\right)$ is a distal extension of $(X, S)$ (under $\left.\varphi_{\alpha}\right)$, then $\left(\times_{\Sigma} X_{\alpha}, S\right)$ is a distal extension of $(X, S)$ (under $\varphi$ ),

(ii) if for each $\alpha \in \Gamma,\left(X_{\alpha}, S\right)$ is a B-distal extension of $(X, S)$ (under $\left.\varphi_{\alpha}\right)$, then $\left(\times_{\Sigma} X_{\alpha}, S\right)$ is a $B$-distal extension of $(X, S)$ (under $\varphi$ ),

(iii) if for each $\alpha \in \Gamma,\left(X_{\alpha}, S\right)$ is a $B \frac{(\bar{M})}{}$ distal extension of $(X, S)$ (under $\varphi_{\alpha}$ ), then $\left(\times_{\Sigma} X_{\alpha}, S\right)$ is a $B \frac{(\overline{\mathrm{M}})}{2}$ distal extension of $(X, S)$ (under $\left.\varphi\right)$.

Proof. (b) (ii) By the definition of $\times_{\Sigma} X_{\alpha}$, we have

$$
\begin{aligned}
\mathrm{R}(\varphi) & =\left\{\left(\left(x_{\alpha}\right)_{\alpha \in \Gamma},\left(y_{\alpha}\right)_{\alpha \in \Gamma}\right) \in\left(\times_{\Sigma} X_{\alpha}\right) \times\left(\times_{\Sigma} X_{\alpha}\right) \mid \forall \alpha \in \Gamma\left(x_{\alpha}, y_{\alpha}\right) \in \mathrm{R}\left(\varphi_{\alpha}\right)\right\} \\
& =\left\{\left(\left(x_{\alpha}\right)_{\alpha \in \Gamma},\left(y_{\alpha}\right)_{\alpha \in \Gamma}\right) \in\left(\times_{\Sigma} X_{\alpha}\right) \times\left(\times_{\Sigma} X_{\alpha}\right) \mid \exists \alpha \in \Gamma\left(x_{\alpha}, y_{\alpha}\right) \in \mathrm{R}\left(\varphi_{\alpha}\right)\right\},
\end{aligned}
$$

moreover, for each $\left(\left(x_{\alpha}\right)_{\alpha \in \Gamma},\left(y_{\alpha}\right)_{\alpha \in \Gamma}\right) \in \mathrm{P}_{\varphi^{-1}(B)}\left(\times_{\Sigma} X_{\alpha}\right)$ and $\gamma \in \Gamma$, we have $\left(x_{\gamma}, y_{\gamma}\right) \in$ $\mathrm{P}_{\varphi_{y}^{-1}(B)}\left(X_{\gamma}\right)$, so if $\left(\left(x_{\alpha}\right)_{\alpha \in \Gamma},\left(y_{\alpha}\right)_{\alpha \in \Gamma}\right) \in \mathrm{R}(\varphi) \cap \mathrm{P}_{\varphi^{-1}(B)}\left(\times_{\Sigma} X_{\alpha}\right)$, then $\left(x_{\gamma}, y_{\gamma}\right) \in \mathrm{R}\left(\varphi_{\gamma}\right) \cap$ $\mathrm{P}_{\varphi_{y}^{-1}(B)}\left(X_{\gamma}\right)$, this will give the desired result, that is, if for each $\alpha \in \Gamma, \mathrm{R}\left(\varphi_{\alpha}\right) \cap$ $\mathrm{P}_{\varphi_{\alpha}^{-1}(B)}\left(X_{\alpha}\right)=\Delta_{X_{\alpha}}$, then $\mathrm{R}(\varphi) \cap \mathrm{P}_{\varphi^{-1}(B)}\left(\times_{\Sigma} X_{\alpha}\right)=\left(\Delta \times_{\Sigma} X_{\alpha}\right)$.

ACKNOWLEDGEMENT. The authors would like to express their appreciation to the referee for his comments and suggestions which improved the original version.

\section{REFERENCES}

[1] R. Ellis, A semigroup associated with a transformation group, Trans. Amer. Math. Soc. 94 (1960), 272-281. MR 23\#A961. Zbl 094.17402.

[2] _ _ Lectures on Topological Dynamics, W. A. Benjamin, New York, 1969. MR 42\#2463. Zbl 193.51502. 
[3] H. Furstenberg, Recurrence in Ergodic Theory and Combinatorial Number Theory, M. B. Porter Lectures, Princeton University Press, New Jersey, 1981. MR 82j:28010. Zbl 459.28023.

[4] M. Sabbaghan and F. A. Z. Shirazi, a-minimal sets and related topics in transformation semigroups (I), Int. Math. Math. Sci 25 (2001), no. 10, 637-654.

Masoud Sabbaghan: DePartment of Mathematics, Faculty of SCIENCE, The UniVERsity of TeHran, Enghelab AVE., TeHran, IRAN

E-mail address: sabbagh@khayam.ut.ac. ir

Fatemah Ayatollah Zadeh Shirazi: Department of Mathematics, Faculty of Science, The University of TeHran, Enghelab Ave., Tehran, Iran

E-mail address: fatemah@khayam. ut.ac. ir 


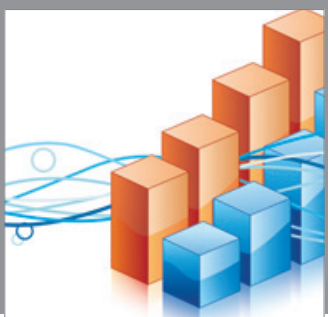

Advances in

Operations Research

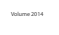

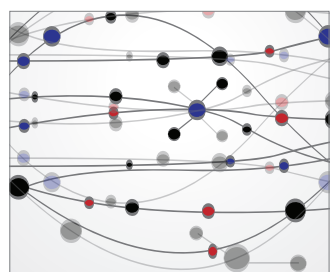

\section{The Scientific} World Journal
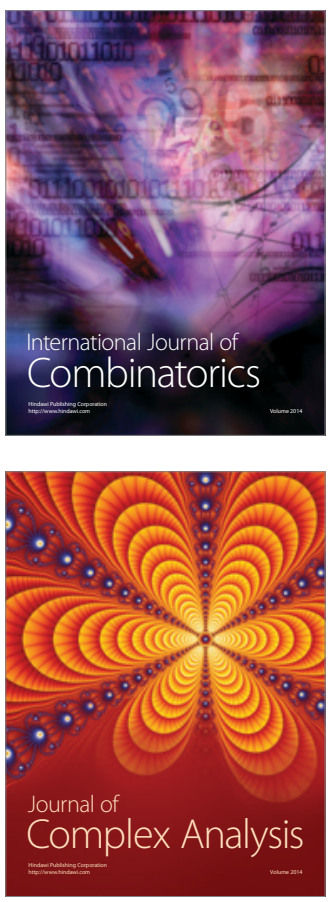

International Journal of

Mathematics and

Mathematical

Sciences
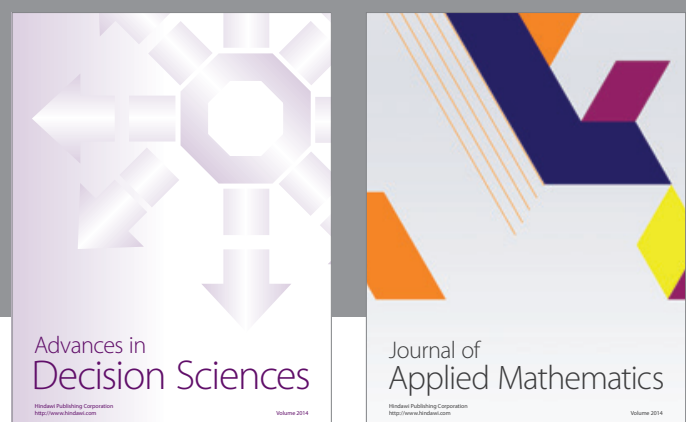

Journal of

Applied Mathematics
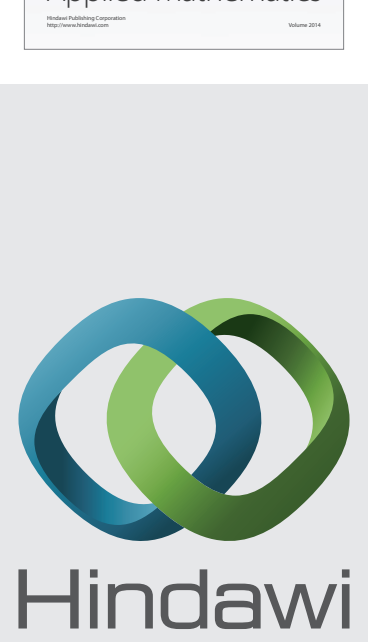

Submit your manuscripts at http://www.hindawi.com
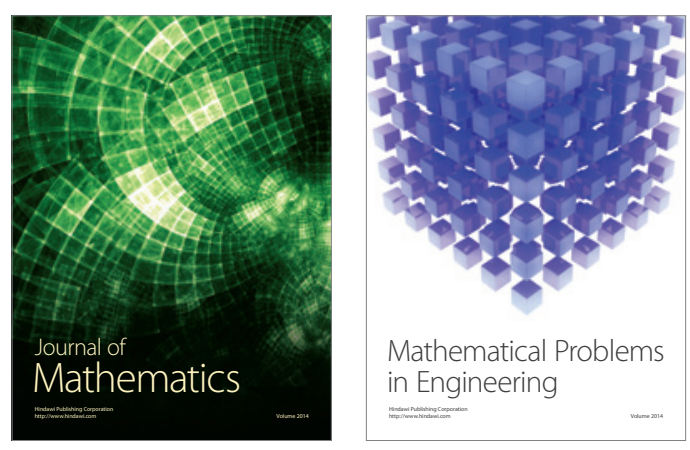

Mathematical Problems in Engineering
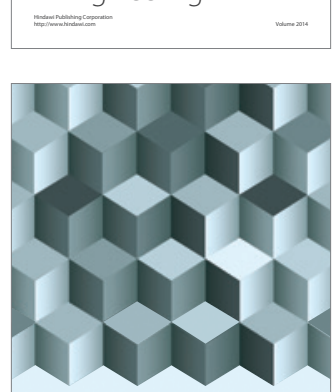

Journal of

Function Spaces
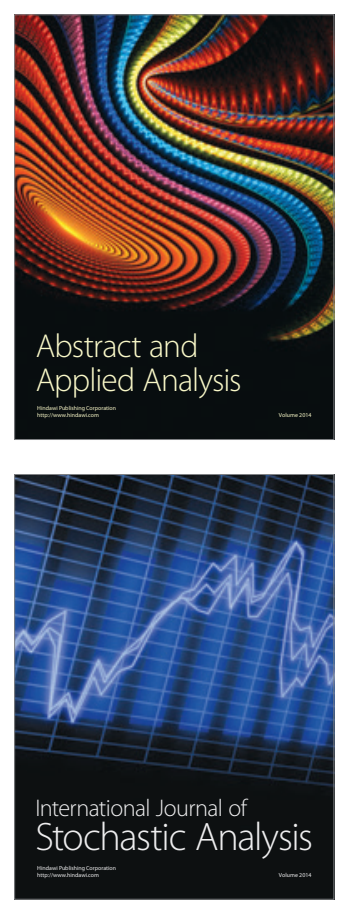

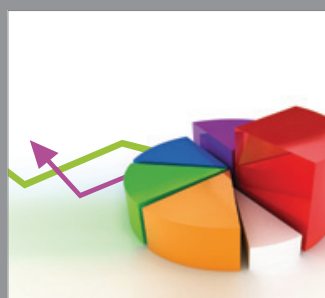

ournal of

Probability and Statistics

Promensencen
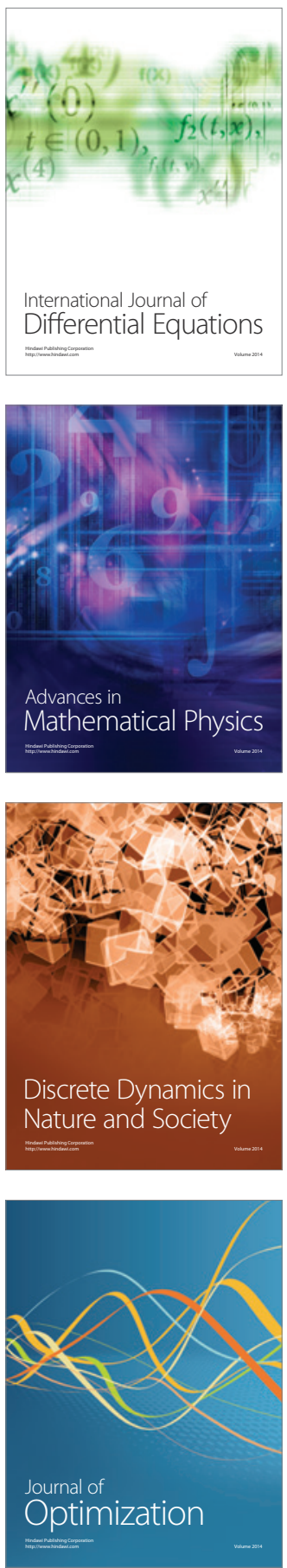